\title{
Transiting exoplanets: From planet statistics to their physical nature
}

\author{
H. Rauer ${ }^{1,2}$
}

${ }^{1}$ Institut für Planetenforschung, DLR, Rutherfordstr. 2, 12489 Berlin, Germany [heike.rauer@dlr.de]

${ }^{2}$ Zentrum für Astronomie und Astrophysik, Technische Universität Berlin, Hardenbergstr. 36, 10623 Berlin, Germany

\begin{abstract}
The colloquium "Detection and Dynamics of Transiting Exoplanets" was held at the Observatoire de Haute-Provence and discussed the status of transiting exoplanet investigations in a 4.5 day meeting. Topics addressed ranged from planet detection, a discussion on planet composition and interior structure, atmospheres of hot-Jupiter planets, up to the effect of tides and the dynamical evolution of planetary systems. Here, I give a summary of the recent developments of transiting planet detections and investigations discussed at this meeting.
\end{abstract}

\section{The increasing planet statistics}

It is fifteen years since the first announcement of an extrasolar planet (51 Peg b) orbiting a solar type star was made (Mayor et al. 1995), based on observations at the Observatoire de Haute-Provence (OHP). This colloquium, focusing on transiting extrasolar planets, follows a colloquium held at OHP in 2005 (Arnold, Bouchy \& Moutou 2006) which was organized to celebrate the anniversary of the detection of 51 Peg. In these last five years not only the detection statistics of exoplanets have increased significantly, but we have also seen the beginning of a new era of exoplanet investigations, addressing the physical properties of planets like their mass-radius relationships, atmospheric composition and orbital evolution. This development is a major step forward towards a meaningful comparison of extrasolar planets to the well-known planets in our Solar System and to a better understanding of planet formation and evolution in general. The recent investigations of the nature of extrasolar planets were made possible by the increasing number of known transiting extrasolar planets. Thus, although hampered by the low geometrical transit probability when considered purely from the viewpoint of detection statistics, the transit method has opened a new window for the investigation and understanding of extrasolar planets.

Figure 1 shows the current (Dec. 1, 2010) statistics of exoplanet detections (from exoplanet.eu), indicating the different methods used. The fraction of known transiting planets has increased significantly since 2005. Most known transiting planets were detected directly by the transit method, but transits are also observed for planets originally detected via radial velocity (rv) measurements. In 2010, about half of the planets were detected by rv and half by transit surveys. Several of these have been presented at this conference. Ground-based transit surveys (e.g. WASP (Hellier et al. and Faedi

This is an Open Access article distributed under the terms of the Creative Commons Attribution-Noncommercial License 3.0, which permits unrestricted use, distribution, and reproduction in any noncommercial medium, provided the original work is properly cited. 


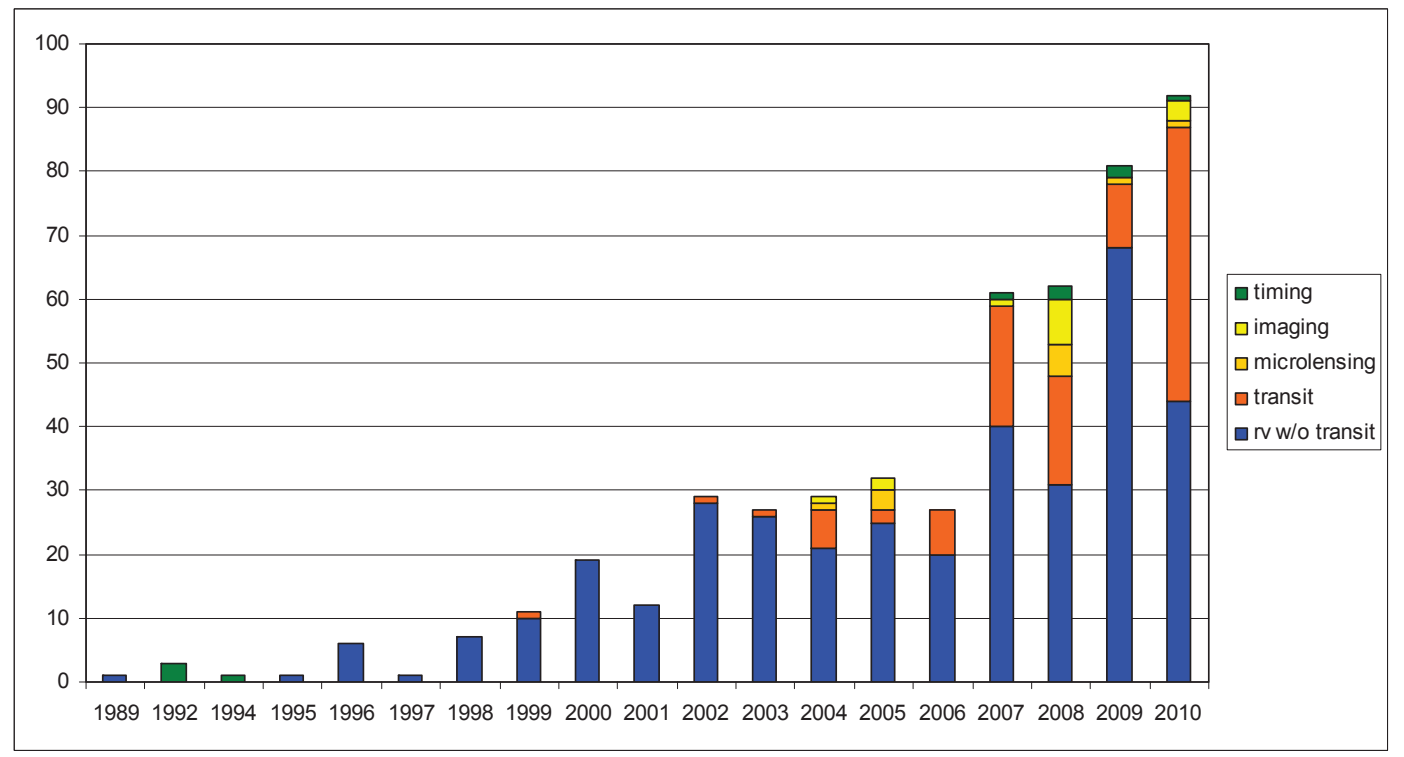

Figure 1: Planet detection history. Blue: planets detected by radial velocity (rv) and without observed transits, red: planets with observed transits (detected by transit or rv), other: detected as indicated. (source: exoplanet.eu, Dec. 1, 2010)

et al., this volume) and HAT (Bakos et al., this volume)) presented 17 new planets. The Kepler team (Holman et al. 2010) discussed 2 transiting planets (Kepler $9 \mathrm{~b}$ and c) orbiting in the same planetary system. These planets show for the first time clear transit timing variations. They also provided indications of a possible third planet on a short period orbit. Kepler-9 d was detected only after the transit timing variation signal from the two planets already-discovered in this system was removed. It is still difficult to rule out all false-positive contaminations, but recent work supports its planet nature even though its mass can not be derived yet (Torres et al. 2011). In 2010, the Kepler-mission released a first list of planet candidates which then triggered the detection of further transiting planets and a white dwarf from rv follow-up of these candidates (Hébrard et al., this volume; Ehrenreich et al. 2010). Furthermore, a planetary system with 7 planets was announced by the rv-team in Geneva (Lovis et al. 2010), including a small super-Earth planet of only $1.4 \mathrm{M}_{\text {Earth }}$ sin i. This non-exhaustive list of recent planet detections presented and discussed at this colloquium illustrates the significant increase in detection statistics made in the past few years. New surveys, like the presented ASTEP400 project located at Antarctica (Crouzet et al., this volume), upgrades of existing surveys and the ongoing and future space missions will provide many further transiting planets for future investigations. 


\section{Planets in the habitable zone?}

The determination of the full planet mass function over all masses and orbital parameters and for all types of central stars is key to understanding planet formation and dynamical evolution. In addition, the detection of small, terrestrial (super-)Earth planets is the subject of special attention in all exoplanet surveys because these planets are potential objects for habitability outside our Solar System. Figure 2 shows the location of the habitable zone (HZ), defined as the orbital distance where liquid water can exist on the surface of a terrestrial planet (Kasting et al. 1993). In our Solar System, only Earth is well within the HZ, whereas Venus and Mars are near its edges and have been habitable only for a short time interval in their evolution if at all.

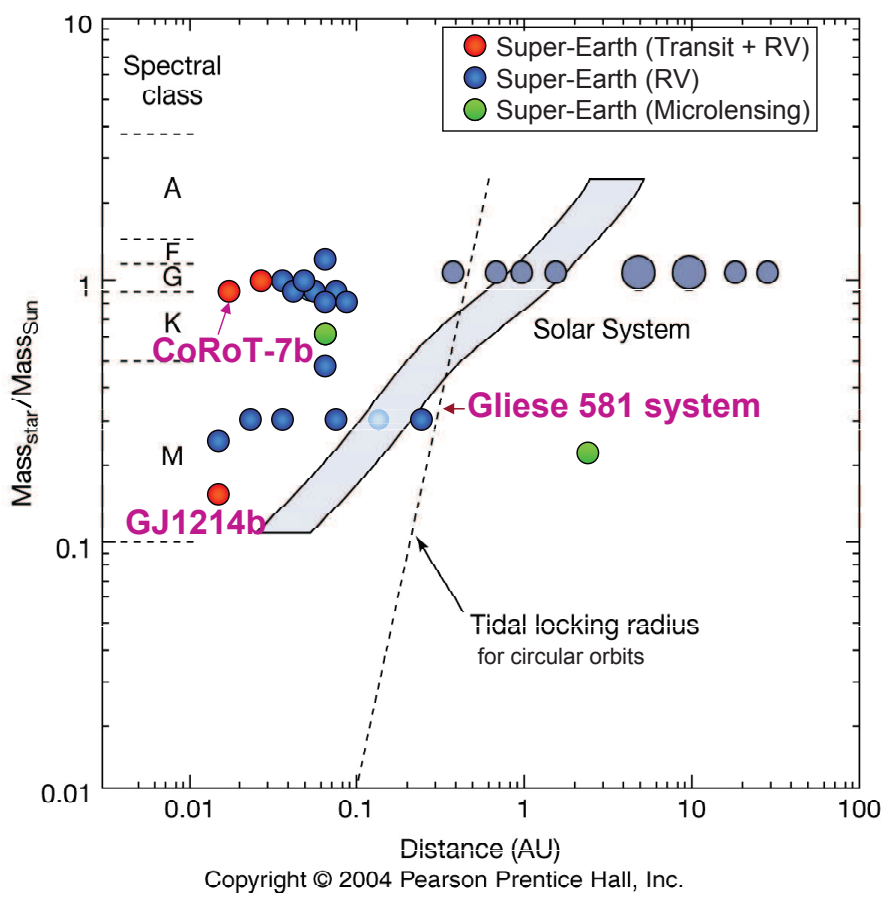

Figure 2: Illustration of the location of the currently known super-Earth planets with respect to the habitable zone as defined by Kasting 1993.

Figure 2 also shows the location of the presently known super-Earth planets i.e. planets with $m$ (sin i) < 10 Earth masses (parameters from exoplanet.eu). The majority of super-Earth planets have been detected within 0.1 AU from their central star. Only one planet, detected by microlensing is found outside $1 \mathrm{AU}$, but since it orbits an $\mathrm{M}$ dwarf star, it is well beyond the corresponding HZ. The super-Earth planets detected by transits surveys up to now (CoRoT-7b, GJ1214 and Kepler-9d) are far too close to their star to be near the HZ. Today, only one exoplanet system (GJ581) has been detected with planets near the HZ. Recent model simulations (von Paris et al. 2010, Wordsworth et al. 2010) showed that GJ581d can indeed provide habitable conditions for certain $\mathrm{CO}_{2}$-rich atmospheres. Thus, one planet in the $\mathrm{HZ}$ is already known. Meanwhile, 


\section{EPJ Web of Conferences}

additional planets have been announced in the GJ581 system, including a planet well within the HZ, GJ581g (Vogt et al. 2010). This planet would indeed show habitable conditions for a much wider range of atmospheric parameters. However, its detection is presently controversial and needs to be confirmed (Pepe, F. 2010; Anglada-Escudé 2011).

Fig. 2 shows that the detection of super-Earth planets in the HZ is difficult up to now, for transits as well as for rv surveys. M dwarf central stars provide the best chances due to the proximity of the HZ to the star in these systems. They, however, may have other difficulties if one considers the harsh radiation environment planets may suffer around these mostly very active stars.

In future, super-Earth detection surveys need to be improved to fill the gap between the location of the $\mathrm{HZ}$ and the current detection range for terrestrial planets. In parallel, theoretical modeling activities are needed to better define the location of the HZ, e.g. for stars with very different activity levels than found in our Solar System.

\section{Detection range of transit surveys}

Figure 3 shows the transit depth $\left(\frac{R_{p}^{2}}{R_{s}^{2}}\right)$ versus the radius of the star, $\mathrm{R}_{s}$, for different planet radii, $\mathrm{R}_{p}$. The figure also shows the presently known transiting planets (black dots). We can see that most detected transiting planets have radii around $1-1.3 \mathrm{R}_{J}$ and orbit stars larger than about $0.7 \mathrm{R}_{s}$. The detected transit depth is between about $0.04 \%$ up to about 4\%, with HAT-11b showing the smallest (so far) detected transit depth from ground-based observations which have resulted in a confirmed planet detection so far. I arbitrarily chose HAT-11b therefore as the approximate empirical detection limit of current ground-based transit surveys (indicated as the green dashed horizontal line). This is not however a strict limit, and we may well soon see smaller detected transit depths as data reduction techniques and observing strategies improve - both for the already-operating surveys, as well as for newly-planned future instruments. Here, it serves as a marker of what appears to be "state-of-the-art" for ground-based transit detection.

Since the transit depth increases for smaller central stars, it is possible to detect much smaller planets around M dwarf stars with the same detection thresholds. We can see examples of such detections with the super-Earth planet GJ1214b and the superNeptune GJ436b. The number of detections around $\mathrm{M}$ dwarf stars with $\mathrm{R}<0.7 \mathrm{R}_{s}$ is, however, still low because they have only recently started to become targets of dedicated transit search strategies (e.g. the MEARTH project (Nutzman \& Charbonneau 2008)). Comparing the green solid lines, which indicate the transit depths of super-Earth and Earth-sized planets with the current empirical detection limit of ground-based surveys, we can see that terrestrial planets may be detected from the ground but currently only for those planets orbiting the smallest M-dwarfs. Of course, surveys of $\mathrm{M}$ dwarf stars also have to overcome the additional difficulty of the much fainter target stars. Nevertheless, this parameter range is highly interesting for future transit searches, because of the close location of the HZ around cool M dwarfs (see Fig. 2).

The blue-dashed horizontal line in Fig. 3 marks the observation of transits of HD149026b. This planet was previously detected by rv-measurements, and the followup transit observations were then made with a larger aperture telescope than usually used for transit detection surveys. I have therefore arbitrarily indicated the region below the blue-dashed line as the domain of space-based surveys. Terrestrial planets around solar-type stars can be detected only from space and seem out of reach for ground-based surveys. The smallest planets in this regime are CoRoT-7b (Léger et al. 


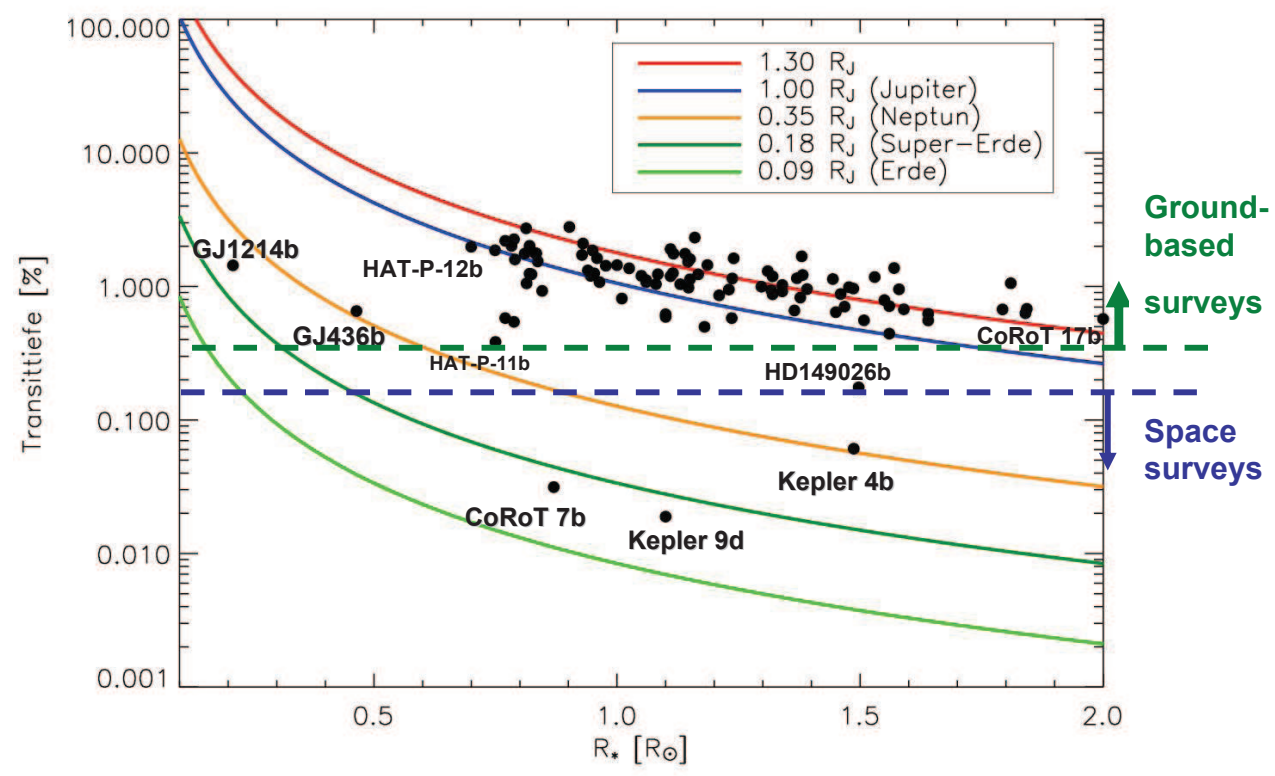

Figure 3: Transit depth versus stellar radius for different planet radii. Black dots show position of known transiting planets.

2009, Queloz et al. 2009) and Kepler-9d (Holman et al. 2010). Both also illustrate the difficulties to confirm such low-mass planets and determine their mass. As discussed below, the mass of CoRoT-7b is still somewhat uncertain, and the mass of Kepler-9d (needed to confirm its detection) has not yet been determined.

The current space missions CoRoT and Kepler as well as future missions proposed in Europe (PLATO) and the U.S. (e.g. TESS, ASTRO) target the detection of small transit depths to fill the known planet mass function at its low mass and radius end and to detect terrestrial planets with known radii and masses for future spectroscopic characterization. For small M dwarfs sensitive space surveys could even detect planets well below Earth size.

\section{Planet basic parameters and spectral characterization}

Planets with observed transits provide a huge potential for the detailed investigation of the objects found. The most surprising discovery in the last few years was made via the Rossiter-McLaughlin (RM) effect. It allows us to determine the angle between the projected rotation axis of the planetary orbit and the stellar spin axis as well as the sense of planet revolution by precise rv-measurements during planet transits. Surprisingly, out of about 30 planets with detected RM effect (Nov. 2010) approximately $30 \%$ showed highly inclined orbits, and some even show evidence for (potentially) retrograde orbits. These findings have stimulated numerous modeling efforts to explain the dynamical evolution of such planets, and were also a focus of this colloquium. In particular the need for improved modeling taking into account the stellar-planet tidal interaction was 
identified and mechanisms of tidal interaction (e.g. the Kozai effect, tidal inflation, etc.) were reviewed (Mardling, this volume). Cébron et al. (this volume) discussed the potential role of elliptical instabilities that might be able to affect the stellar rotation axis, thereby providing another mechanism that could explain the observed inclined systems.

The combination of radii derived from transits and masses from rv-measurements allows us to place a planet in modeled mass-radius diagrams and to determine its mean density. While this is possible with good accuracy for Jupiter and Neptune-sized planets, it is however still challenging for terrestrial objects. An example discussed at this conference, illustrating the state-of-the-art in determining the basic planet parameters of terrestrial planets, is CoRoT-7b. Its derived radius and mass was updated several times since the detection announcement (Léger et al. 2009, Queloz et al. 2009) due to revised stellar parameters (Bruntt et al. 2010) and the potential presence of a third planet in the rv-data (Hatzes et al. 2010). At this meeting, the additional effect of stellar activity in the radial velocity data analysis was discussed (Pont, Aigrain \& Zucker 2010). A recent independent re-analysis of the rv-data increased the mass range for CoRoT-7b again (Ferraz-Mello et al. 2010, Boisse et al. 2010). While the planet radius derived from the transit measurement now seems well constrained subsequent to revising the stellar parameters, the published range of planet masses range from a small terrestrial planet up to a large super-Earth. This ongoing discussion shows that terrestrial planet detections around a relatively faint star such as CoRoT-7b (V=11.7 mag) are near our current limit of observations and data reduction. The discussion, however, gives clear guidelines for future transit and rv-surveys, namely to focus on much brighter target stars where such controversies can be solved easier. In addition, the rv follow-up strategies have to be optimized for such controversial cases, for example by simultaneous rv- and photometric measurements.

Once radius and mass are well known, they can be used to constrain the interior structure of planets. For hot-Jupiter planets the detected radii span a wide parameter range for similar masses. For the large, inflated planets, this observation still remains to be fully explained by theory. The investigation of mass-radius relationships of terrestrial planets has just begun (e.g. Valencia et al. 2010, Wagner et al. 2010). Whether a similar spread in basic planet parameters (radius and mass) is also found still needs to be determined by increasing the number of known transiting terrestrial planets.

Transiting planets furthermore provide the opportunity for spectroscopic characterization of their atmospheres. Observations during primary and secondary eclipses provide data on the planetary atmosphere. Orbital phase variations due to reflected light have also been detected. Such characterizing observations are usually made at low resolution. Today, they are restricted to hot-Jupiter and Neptune sized planets around the brightest host stars. Future space telescopes aim to reduce this limit to include super-Earth planets in such spectroscopic studies.

\section{Summary}

In summary, this colloquium has shown that scientific investigations of extrasolar planets have entered a new phase in recent years, from pure detection statistics to investigations of the physical nature of planets. This development is expected to continue over the years to come with the help of new observing facilities from ground and space, e.g. the ELT, JWST, proposed future transit missions, like PLATO, TESS, ASTRO, but also planets detected by other methods (e.g. direct detection) which will give access to an expanded parameter range. 


\section{Detection and Dynamics of Transiting Exoplanets}

\section{References}

Anglada-Escud, G., 2010, submitted to ApJL, arXiv:1011.0186

Arnold, L., Bouchy, F. \& Moutou, C., 2006, Tenth Anniversary of 51 Peg-b: Status of and prospects for hot Jupiter studies. Colloquium held at Observatoire de Haute Provence, France, August 22-25, 2005

Boisse, I.; Bouchy, F.; Hébrard, G.; et al., 2010, arXiv:1012.1452

Bruntt, H.; Deleuil, M.; Fridlund, M.; et al., 2010, A\&A, 519

Ehrenreich, D.; Lagrange, A.-M.; Bouchy, F.; et al., 2010, A\&A, 525

Ferraz-Mello, S.; Tadeu dos Santos, M.; Beauge, C.; Michtchenko, T. A.; Rodriguez, A., 2010, arXiv:1011.2144

Hatzes, A. P.; Dvorak, R.; Wuchterl, G.; et al., 2010, A\&A, 520

Holman, M.J., Fabrycky, D.C., Ragozzine, D., et al., 2010, Science 330, 51

Kasting, J.F., Whitmire, D, P., Reynolds, R. T., 1993, Icarus 101, 108-128

Léger, A.; Rouan, D.; Schneider, J.; et al., 2009, A\&A, 506, 287-302

Lovis, C., Ségransan, D., Mayor, M., et al., 2010, eprint arXiv:1011.4994

Mayor, M., \& Queloz, D. 1995, Nature 378, 355-359

Nutzman, P., \& Charbonneau, D. 2008, PASP, 120, 317

Pepe, F. 2010, IAU Symposium : The Astrophysics of Planetary Systems. Oral Contribution

Pont, F., Aigrain, S., Zucker, S., 2010, MNRS accepted, arXiv:1008.3859

Queloz, D.; Bouchy, F.; Moutou, C.; et al., 2009, A\&A, 506, 303-319

Torres, K.B.V., Lampens, P., Frmat, Y., Hensberge, H., Lebreton, Y., koda, P., 2011, A\&A525

Valencia, D.; Ikoma, M.; Guillot, T.; Nettelmann, N., 2010, A\&A, 516

Vogt, S. S., Butler, R. P., Rivera, E. J., et al., 2010, ApJ, 723, 954

von Paris, P., Gebauer, S., Godolt, M., et al., 2010, A\&A522

Wagner, F. W.; Sohl, F.; Rauer, H.; Hussmann, H.; Grott, M., 2010, Highlights of Astronomy, 15, 708-709

Wordsworth, R. D., Forget, F., Selsis, F., Madeleine, J.-B., Millour, E., Eymet, V., 2010, A\&A522, 